\title{
On Discernibility and Symmetries
}

\author{
Tomasz Bigaj
}

Received: 23 November 2013/ Accepted: 12 March 2014/Published online: 26 March 2014

(C) The Author(s) 2014. This article is published with open access at Springerlink.com

\begin{abstract}
This paper addresses the issue of the multiplicity of various grades of discernibility that can be defined in model theory. Building upon earlier works on the subject, I first expand the known logical categorizations of discernibility by introducing several symmetry-based concepts of discernibility, including one I call "witness symmetry-discernibility". Then I argue that only grades of discernibility stronger than this one possess certain intuitive features necessary to individuate objects. Further downsizing of the set of non-equivalent grades of discernibility can be achieved by stipulating that any relation of discernibility should be applied only to those pairs of objects which have been previously distinguished (in a suitable sense) from the rest of the universe. Restricting discernibility to pairs of objects satisfying this condition gives an additional bonus in the form of restoring the transitivity of some types of indiscernibility which have been known to be non-transitive.
\end{abstract}

\section{Introduction}

The notion of qualitative discernibility is extensively used in modern metaphysical discussions on the topic of identity and individuality. Recent foundational work has revealed that the concept of discernibility admits a whole spectrum of possible formal interpretations. ${ }^{1}$ There are several known general types, or grades, of discernibility, and they in turn split into further subcategories

\footnotetext{
${ }^{1}$ The most comprehensive analyses of the logic of discernibility to date include (Ketland 2011; Caulton and Butterfield 2012; Ladyman et al. 2012).
}

T. Bigaj ( ()

Department of Philosophy, University of California, San Diego, CA, USA

e-mail: t.f.bigaj@uw.edu.pl; tfbigaj@gmail.com

T. Bigaj

Institute of Philosophy, University of Warsaw, Warsaw, Poland 
depending on the expressive power of the language in which the discerning is done. The abundance of various interpretations of discernibility is troubling, since it seriously hampers any constructive debate involving this notion, the reason being that for each argument using one interpretation of discernibility an equally compelling counterargument employing an alternative interpretation is likely to be found. In particular, questions such as "Are elementary particles (or spacetime points) discernible?" do not admit definite answers, since it is necessary to specify further what sense of discernibility the inquirer has in mind. ${ }^{2}$ While in philosophy it is not uncommon to give only qualified and conditional answers to many important questions, some measures might be considered to reduce the plethora of logical concepts brought together under the umbrella of discernibility.

I believe that this goal can be achieved by reconsidering what work the concept of discernibility is supposed to do for us. When we talk about discerning two objects, we usually have one of the following two separate things in mind. One sense of discerning involves recognizing some qualitative differences (whether in the form of different properties or different relations) between the objects considered. When we discern objects in this sense, we should (at least in principle) be able to pick out one of them but not the other. Being able to discern objects in that way seems to be a prerequisite for making successful reference, or giving a unique name, to each individual object. But by discerning we can also mean recognizing objects as numerically distinct. In this sense of the word, discernment is a process by which, using some qualitative features of the objects, we make sure that there are indeed two entities and not one.

The latter of the above-mentioned intuitions associated with discernibility is related to the much-discussed metaphysical goal of "grounding" numerical distinctness in qualitative facts. The rough idea behind this goal is that the fact that object $a$ is numerically distinct from object $b$ cannot be "bare", or further unanalyzable. There must be some qualitative facts about $a$ and $b$ which "make" them two objects rather than one. Thus, two objects are discerned in the considered sense if there is some fact involving their qualitative properties and relations which logically entails that they are numerically distinct. But numerical diversity does not necessarily guarantee that the objects in question will achieve the metaphysical status of individuals. Individuation involves things such as the possibility of

\footnotetext{
2 The impact of the existence of multiple interpretations of discernibility on some debates in modern metaphysics of science can be best illustrated using the problem of the identity and individuality of particles in quantum mechanics as an example. The symmetrization postulate, which is an important part of the formalism of the quantum theory of many particles, is taken to imply that particles of the same type are not discernible by properties and relations, and thus violate the Leibnizian Principle of the Identity of Indiscernibles (French and Redhead 1988, Butterfield 1993). However, some authors argue that there is a sense of discernibility (known as weak discernibility) which allows a variant of the PII to retain its validity for identical fermions and even bosons (Saunders 2003, 2006; Muller and Saunders 2008; Muller and Seevinck 2009). Similar arguments have also been used with respect to spacetime points (see e.g. Wüthrich 2009).
} 
reference, or labelling, and therefore is closely associated with the first of the above senses of discernibility. ${ }^{3}$

Throughout this paper I will limit myself solely to the first reading of the notion of discernibility. I believe that the recent discussions on the discernibility of objects postulated in contemporary physical theories are mainly motivated by the need to ensure that these objects are indeed individuals, and not by the desire to ground their numerical diversity in qualitative facts. I will argue that only a small subset of all the available grades of discernibility can actually accomplish the former task, and this observation can lead to a significant reduction of their number, as will be shown in Sects. 3 and 5. Moreover, this number can be further scaled down by introducing a new restriction on the pairs of objects that are supposed to be discerned. This issue will be discussed in Sects. 6 and 7 of this paper. Sections 2 and 4 will mostly contain summaries of known facts regarding extant grades of discernibility and their connections with the presence of symmetries.

\section{Grades of Discernibility}

We will start our investigations by giving a brief overview of the known grades of discernibility and their logical relations (in this exposition I will closely follow Ladyman et al. 2012). Let $\mathcal{L}_{A}$ be a first-order language without the identity symbol and without constants, which describes a particular relational structure $\mathcal{A}$. The following standard types of discernibility (originally due to Quine 1976) are defined as follows:

(1) Absolute discernibility: $\operatorname{Abs}_{A}(a, b)$ iff there is a formula $\varphi(x)$ in $\mathcal{L}_{A}$ such that $\mathcal{A} \vDash \varphi(a)$ and $\mathcal{A} \not \models \varphi(b)$.

(2) Relative discernibility: $\operatorname{Rel}_{A}(a, b)$ iff there is a formula $\varphi(x, y)$ in $\mathcal{L}_{A}$ such that $\mathcal{A} \vDash \varphi(a, b)$ and $\mathcal{A} \not \models \varphi(b, a)$.

(3) Weak discernibility: $\operatorname{Weak}_{A}(a, b)$ iff there is a formula $\varphi(x, y)$ in $\mathcal{L}_{A}$ such that $\mathcal{A} \vDash \varphi(a, b)$ and $\mathcal{A} \not \models \varphi(a, a)$.

It is sometimes useful to distinguish as a separate category intrinsic discernibility $\operatorname{Int}_{A}(a, b)$, which is just absolute discernibility restricted to formulas that do not contain any quantifiers. Two more languages can be considered besides $\mathcal{L}_{A}$ : one is the language $\mathcal{L}_{A}^{=}$containing identity, and the other is the language $\mathcal{L}_{A *}$ which is assumed to contain an individual constant for each element in the domain of model $\mathcal{A}$ (technically this means that $\mathcal{L}_{A *}$ describes not structure $\mathcal{A}$ but its extension $\mathcal{A}^{*}$ in which all elements of the domain are distinguished). Definitions 1-3 can now be repeated with languages $\mathcal{L}_{A}^{=}$and $\mathcal{L}_{A *}$ replacing $\mathcal{L}_{A}$, and this gives us six more grades of discernibility: $\operatorname{Abs}_{A}^{\overline{\bar{A}}}(a, b), \operatorname{Rel}_{A}^{\bar{E}}(a, b), \operatorname{Weak}_{A}^{\bar{*}}(a, b), \operatorname{Abs}_{A^{*}}(a, b), \operatorname{Rel}_{A^{*}}(a, b)$,

\footnotetext{
3 Muller and Saunders (2008), as well as Caulton and Butterfield (2012), use the term "individual" as referring to objects that are absolutely discernible. While absolute discernibility guarantees the possibility of individuation in the above sense, later I will argue that there may be other types of discernibility that achieve the same objective. Throughout the paper I will use the term "individual" in a sense broader than the one used by the above-mentioned authors. I urge the reader to keep this in mind, to avoid possible confusion.
} 
Weak $_{A *}(a, b)$. The logical relations between the introduced variants of discernibility are depicted in Fig. 1 below (cf. Ladyman et al. 2012, p. 177). ${ }^{4}$

From the diagram we can immediately notice that there are actually 7 extensionally distinct grades of discernibility (or 5 if we ignore intrinsic variants of absolute discernibility), which is a rather large number. It is easy to observe that each variant of discernibility can play the role of a qualitative "grounding" of the relation of numerical distinctness, since all of them logically imply that $a \neq b .{ }^{5}$ However, it is not at all clear whether all notions of discernibility, when applied to objects $a$ and $b$, can be assumed to ensure that $a$ is qualitatively different from $b$ in the sense specified above. We will now move on to discuss this issue in more detail.

\section{Witness-Discernibility}

How can we single out one specific object out of a pair of entities using their purely qualitative characteristics? One sure method to do that is to appeal to a difference in properties. If $a$ possesses a property $P$ which $b$ lacks, this can give us a simple method of separating them as individual entities. In such a case we can always refer to $a$ and not to $b$ with the help of the description "The object that possesses $P$ ". Thus, absolute discernibility (whether intrinsic or extrinsic) clearly achieves the required objective.

\footnotetext{
${ }^{4}$ It is also possible to consider the language $\mathcal{L}_{A *}^{=}$containing both the identity symbol and constants for all elements of the domain. However, it is easy to observe that all grades of discernibility in $\mathcal{L}_{A *}^{=}$collapse into numerical distinctness, therefore I will ignore this possibility. The issue of which of the three languages $\mathcal{L}_{A}, \mathcal{L}_{A}^{=}$, and $\mathcal{L}_{A *}$ should be preferred in discussions on the concept of discernibility is a delicate one, and I do not wish to make any definite assertion here, noting only some typical arguments and counterarguments that may be used in the debate. First, it may be pointed out that the practice of using the identity symbol is so widespread in any scientific language that the language $\mathcal{L}_{A}$ seems to be unrealistically impoverished. Nonetheless, in the context of discernibility there may be good reasons for holding on to such an artificially restricted language. As is well known, in language with identity all numerically distinct objects are weakly discernible by the formula $x \neq y$. Hence those who believe that the weak discernibility of elementary particles or spatiotemporal points restores their metaphysical status of individuals (or at least that of relationals, if we adhere to Muller and Saunders's terminology) had better not use the language with identity on pain of trivializing their claim. Second, there are good reasons to remain skeptical regarding the utility of the language $\mathcal{L}_{A *}$, which contains names for each individual in the domain. But there are two interesting logical facts about discernibility in $\mathcal{L}_{A *}$ which are worth mentioning here. One is that, contrary to popular belief, distinct objects are not absolutely discernible in $\mathcal{L}_{A *}$ by virtue of possessing their unique names (we need identity to construct absolutely discerning formulas in $\mathcal{L}_{A *}$ ). The other is that absolute, relative and weak discernibilities become extensionally equivalent in $\mathcal{L}_{A *}$. However, given the limited applicability of $\mathcal{L}_{A *}$ in reconstructing scientific theories, I will mostly ignore it in further considerations.

${ }^{5}$ On the other hand, it should be stressed that the implications in the opposite direction (i.e. from the numerical distinctness of objects to their discernibility of appropriate kinds) are not guaranteed by logic alone. In order to close the logical gap between discernibility and numerical distinctness we need a metaphysical assumption in the form of the Principle of the Identity of Indiscernibles (PII). As I already noted in footnote 2 , the recent interest in developing new grades of discernibility weaker than absolute discernibility has been spurred by the apparent violation of the absolute form of PII in the case of quantum particles of the same type and the case of space-time points (see also my closing remarks at the end of Sect. 8).
} 


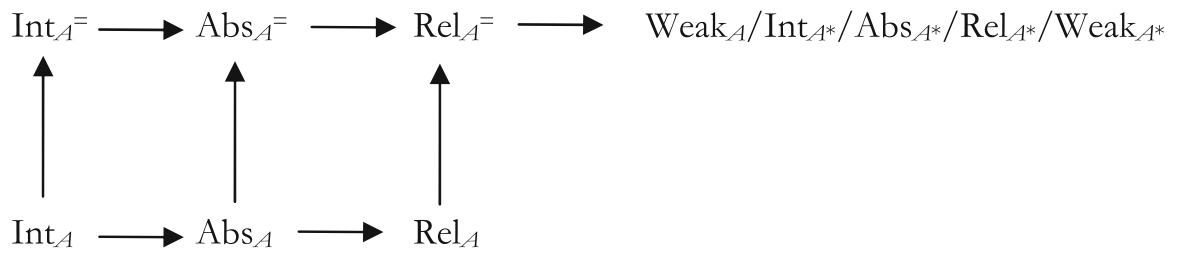

Fig. 1 Logical relations between grades of discernibility

However, this is not the only method of differentiating between objects. Even if $a$ and $b$ are not discernible by their properties, still it may be possible to tell them apart using other objects as reference points. It may happen, for instance, that there is yet another object $c$ in the domain for which it is true that $c$ stands in a certain relation $R$ to $a$ but not to $b$. Such an object can be called a witness, and the existence of a witness (henceforth referred to as witness-discernibility) seems to secure the possibility of an effective separation of $a$ and $b$.

Using the generic concept of witness-discernibility, it may now be argued that even merely weakly discernible objects can be effectively differentiated by a witness. For if $\varphi(x, y)$ is a formula which weakly discerns $a$ and $b$, then it is true that there is an object $c$ such that $\mathcal{A} \models \varphi(c, b)$ and yet $\mathcal{A} \not \models \varphi(c, a)$-it is namely $a$ itself. However, we can immediately notice that something is not right here. How can an object which has not yet been differentiated from the other one play the role of a witness to do the differentiation? Clearly, some further restrictions have to be placed on the notion of a witness. One obvious candidate for such a requirement seems to be that a witness should not be identical with any of the two objects to be discerned by it. But this restriction is too weak, as the following graph shows (Fig. 2). ${ }^{6}$

Here $c$ apparently assumes the role of a witness for $a$ and $b$, since it stands in some relation $R$ (represented by an arrow) to $a$ and not to $b$. But an effective identification of $a$ by $c$ is thwarted by the existence of yet another object $d$ which stands in the same relation to $b$ and not to $a$. Since $c$ and $d$ do not bear any labels that may differentiate them, it is impossible to say which of them is supposed to be our selected witness. This idea was picked up by Ladyman and Bigaj (2010) in their proposal of how to define a satisfactory concept of witness-discernibility (which they call "relational physical discernibility"). They insist that for an object to be a witness it has to be the case that all objects not absolutely discernible from it should remain in the same relation $R$ to $a$ and not to $b$. Thanks to this additional requirement it doesn't actually matter which object in a given absoluteindiscernibility class we will select to serve as a witness-all of them will uniformly point to $a$ and not to $b$. Ladyman and Bigaj's concept of witness discernibility can be spelled out as follows:

\footnotetext{
${ }^{6}$ Actually, this restriction is also too strong. In some cases it is legitimate to use one of the differentiated objects as a witness. This is the case when objects $a$ and $b$ are absolutely discernible. In that case object $a$ can be considered a witness discerning $a$ from $b$.
} 


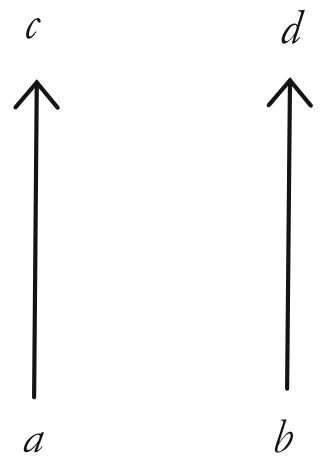

Fig. 2 A scenario in which objects are not discerned by a witness

(4) Witness discernibility: $\operatorname{Wit}_{A}(a, b)$ iff there is an object $c \in \operatorname{dom}(\mathcal{A})$ ) and a formula $\varphi(x, y)$ in $\mathcal{L}_{A}$ such that for all $d \in \operatorname{dom}(\mathcal{A})$, if $\neg \operatorname{Abs}_{A}(c, d)$, then $\mathcal{A} \vDash \varphi(d, a)$ and $\mathcal{A} \not \models \varphi(d, b){ }^{7}$

As was the case with the other grades of discernibility, witness discernibility can be alternatively defined relative to a language with identity $\left(\mathcal{L}_{A}^{=}\right)$or a language equipped with constants for each object $\left(\mathcal{L}_{A *}\right)$. The issue of the logical relations between witness-discernibility and the remaining grades of discernibility has been recently scrutinized by Linnebo and Muller (2013). They have proven that witnessdiscernibility actually comes very close to absolute discernibility. More specifically, witness-discernibility is equivalent to absolute discernibility in models with finite domains, as well as in languages that admit infinite formulas. The equivalence also holds relative to languages in which all elements of the domain (whether finite or infinite) have unique names. The only case in which two objects can be witnessdiscerned but not absolutely discerned is when the model contains an infinite number of elements and the language neither allows for infinite conjunctions nor contains names for each individual object.

The effective collapse of witness-discernibility into absolute discernibility is not necessarily bad news for those who are troubled by the excessive number of available interpretations of what it means to discern two objects. For it suggests that our intuitive reading of discernibility as a way to tell two objects apart is best expressed in the good old notion of absolute discernibility by properties (intrinsic or extrinsic). In particular, Ladyman and Bigaj employed their concept of witnessdiscernibility to argue against the use of weak discernibility as a means to rehabilitate the principle of the identity of indiscernibles in the context of quantum mechanics. ${ }^{8}$ But it turns out that the general idea of introducing witnesses to discern

\footnotetext{
7 This definition of witness-discernibility is a simplified but equivalent version of Definition 5 given in (Ladyman and Bigaj 2010, p. 128), following the suggestion made in (Linnebo and Muller 2013, p. 1136).

${ }^{8}$ Ladyman and Bigaj thereby join a long list of critics of weak discernibility which includes, among others, Hawley (2006, 2009), French and Krause (2006), van Fraassen and Peschard (2008) and Dieks and Versteegh (2008). Of course the list of supporters of weak discernibility is equally impressive. Besides Saunders, Muller and Seevinck, who were already mentioned in footnote 2, it contains Caulton and Butterfield (2012), Dorato and Morganti (2013), Huggett and Norton (2013).
} 
objects can find an alternative formalization which is not tied to absolute discernibility. In order to explain this issue in detail, we will have to introduce the important concept of symmetry.

\section{Symmetries}

It is commonly acknowledged that the existence of symmetries is related to the problem of discernibility, although opinions vary as to how close this relation is. ${ }^{9}$ An important difference between discernibility and symmetries is that the former always involves a language, whereas symmetries characterize structures directly without any linguistic mediation. However, we should not forget that there is a close correspondence (even though not one-one) between a structure and a language it is described in. By changing the language in which we wish to discern objects we usually select a different corresponding structure (for instance, adding the identity symbol "=" to our language we have to expand the considered structure to include the relation of identity).

A symmetry of a relational structure $\mathcal{A}$ (also referred to as an automorphism of $\mathcal{A})$ is characterized informally as a bijective mapping of its domain $\operatorname{dom}(\mathcal{A})$ which preserves all its relations. A formal definition is as follows:

(5) A bijection $\pi: \operatorname{dom}(\mathcal{A}) \rightarrow \operatorname{dom}(\mathcal{A})$ is a symmetry of $\mathcal{A}$ iff for every relation $R$ in $\mathcal{A}$ and all objects $a_{1}, \ldots, a_{n} \in \operatorname{dom}(\mathcal{A}), R a_{1} \ldots a_{n}$ iff $R \pi\left(a_{1}\right) \ldots \pi\left(a_{n}\right)$.

Let us define a binary relation $\operatorname{Sym}_{A}$ on the domain of $\mathcal{A}$ :

$\operatorname{Sym}_{A}(a, b)$ iff there is a symmetry $\pi$ of $A$ such that $\pi(a)=b$.

It is easy to observe that $\mathrm{Sym}_{A}$ is an equivalence relation (it is reflexive, symmetric and transitive). Thus, the entire domain can be partitioned into equivalence classes with respect to $\operatorname{Sym}_{A}$. We will call them "classes of symmetry-indiscernibility". Note also that adding the relation of identity to structure $\mathcal{A}$ does not change its symmetries, i.e. $\operatorname{Sym}_{A}(a, b)$ iff $\operatorname{Sym}_{A}^{=}(a, b)$. On the other hand, adding constants for each object in the domain has the effect of reducing all symmetries of relational structure $\mathcal{A}^{*}$ to the trivial one: $\operatorname{Sym}_{A^{*}}(a, b)$ iff $a=b$. For that reason we will only consider one relation of symmetry-indiscernibility $\operatorname{Sym}_{A}(a, b)$ in subsequent discussions.

Now I will report some well-known model-theoretic facts which concern the logical relations between symmetries and other grades of discernibility. ${ }^{10}$

\footnotetext{
9 Ladyman et al. (2012, p. 181) express the opinion that the connection between symmetries and discernibility is not as close as one might expect. But their main argument is that the non-existence of certain symmetries is not equivalent to any previously introduced grades of discernibility. However, it may still be maintained that the lack of certain symmetries defines new grades of discernibility which are logically related to the other grades.

${ }^{10}$ As Ladyman et al. (2012) point out, Theorem 1 follows directly from the fact that symmetries preserve satisfaction of all complex formulae, i.e. if a formula is satisfied by an n-tuple of objects, it will be satisfied by the images of these objects under any symmetry.
} 


\section{Theorem 1}

(a) If $\operatorname{Abs}_{A}(a, b)$, then $\neg \operatorname{Sym}_{A}(a, b)$,

(b) If $\operatorname{Abs}_{A}^{=}(a, b)$, then $\neg \operatorname{Sym}_{A}(a, b)$.

Theorem 1 states that if there is a formula satisfied by one object but not the other (whether the formula contains identity or not), these two objects cannot be connected by a symmetry. However, the question of implication in the opposite direction is more complicated, as the following theorems show.

\section{Theorem $2^{11}$}

(a) If $\mathcal{A}$ is finite, then $\operatorname{Abs}_{A}^{\overline{=}}(a, b)$ iff $\neg \operatorname{Sym}_{A}(a, b)$,

(b) There are infinite structures such that for some $a, b \in \operatorname{dom}(\mathcal{A}), \neg \operatorname{Sym}_{A}(a, b)$ and $\neg \operatorname{Abs}_{A}^{=}(a, b)$.

Theorem 3 There are finite structures such that for some $a, b \in \operatorname{dom}(\mathcal{A})$, $\neg \operatorname{Sym}_{A}(a, b)$ and $\neg \operatorname{Abs}_{A}(a, b)$.

Theorem 2(a) states that in finite models the non-existence of a symmetry connecting two objects $a$ and $b$ is equivalent to absolute discernibility by formulas containing identity. However, according to Theorem 3 even in finite cases it is possible to find objects $a$ and $b$ that are not symmetry-related, and yet no formula without identity can discern them. The following example illustrates such a situation. In this graph there is no symmetry mapping $a$ into $b$, and yet there is no formula without identity which is true of $a$ and false of $b$, since there is no way to express in $\mathcal{L}_{A}$ the fact that $a$ is related to two objects rather than one (Fig. 3).

I do not wish to take a stand on the issue of the admissibility of the identity symbol in discerning formulas. The conventional wisdom is that the use of identity in that context should be avoided on pain of circularity. However, it may be observed that if discernibility is taken as a means to tell two objects apart rather than as a concept grounding numerical distinctness, there seems to be nothing wrong in using identity in discerning formulas. On the other hand, combining identity with constants can have a detrimental effect on the task of discerning objects, since in that case every two objects are trivially discernible by their respective "haecceities" expressed in formulas $x=c$.

The logical relation between symmetry and relative discernibility is given in the following theorem. Theorem 4(a) follows immediately from Theorem 2(a) and the fact that $\mathrm{Abs}_{A}^{=}$implies $\operatorname{Rel}_{A}^{\bar{\nu}}$. An example illustrating point (b) is given in (Ladyman et al. 2012, p. 182).

\section{Theorem 4}

(a) If $\mathcal{A}$ is finite, then $\neg \operatorname{Sym}_{A}(a, b)$ implies $\operatorname{Rel}_{A}^{\bar{A}}(a, b)$

(b) There are infinite structures such that for some $a, b \in \operatorname{dom}(\mathcal{A}), \neg \operatorname{Sym}(a, b)$ and $\neg \operatorname{Rel}_{A}^{=}(a, b)$.

The equivalence classes of symmetry-indiscernibility induced by the set of all automorphisms of a given structure $\mathcal{A}$ can contain varying numbers of elements. If a

\footnotetext{
11 Theorem 2 is proven e.g. in (Caulton and Butterfield 2012, section 4.3).
} 


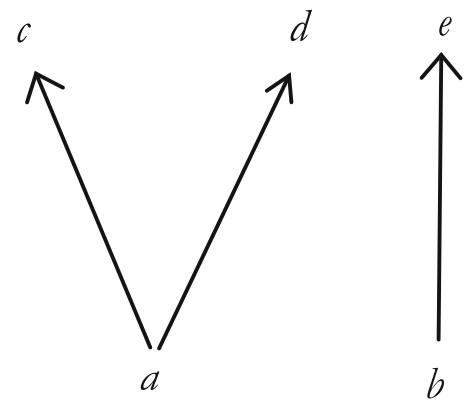

Fig. 3 An example proving Theorem 3

given equivalence class contains only two elements, this implies that there is a symmetry which exchanges these elements: $\pi(a)=b$ and $\pi(b)=a$. However, there is no implication in the opposite direction. From the fact that there is a symmetry which transposes $a$ and $b$ it does not follow that $a$ and $b$ form their own equivalence class. The diagram below illustrates such a situation. Even though the transposition $\pi_{a b}$ (the mapping exchanging $a$ with $b$ and leaving everything else intact) is a symmetry, there is yet another symmetry which exchanges $a$ with $c$ and simultaneously $b$ with $d$. It is easy to observe that in this case all four elements form one class of symmetry-indiscernibility (Fig. 4).

It may be instructive to observe that the relation $\mathrm{Sym}_{A}$ is not the only relation of indiscernibility that may be defined purely in terms of symmetry mappings. Two more variants of symmetry-based indiscernibility, which turn out not to be extensionally equivalent to $\mathrm{Sym}_{A}$, can easily be introduced. ${ }^{12}$ A stronger sense of symmetry-based indiscernibility is obtained by requiring that there be a symmetry which transposes elements $a$ and $b$. Let us write that (the prefix $\mathrm{S}$ stands for "strong"):

(7) $\operatorname{S}_{-} \operatorname{Sym}_{A}(a, b)$ iff there is a symmetry mapping $\pi$ of $\mathcal{A}$ such that $\pi(a)=b$ and $\pi(b)=a$.

Yet another, even stronger type of symmetry-indiscernibility can be defined as follows (SS stands for "super-strong”):

(8) $\operatorname{SS}_{-\operatorname{Sym}_{A}}(a, b)$ iff $\pi_{a b}$ is a symmetry of $\mathcal{A}$.

There are obvious logical relations between the three types of symmetryindiscernibility which can be presented in the form of the following implications: $\operatorname{SS}_{-\operatorname{Sym}_{A}}(a, b) \Rightarrow \operatorname{S}_{-} \operatorname{Sym}_{A}(a, b) \Rightarrow \operatorname{Sym}_{A}(a, b)$. The reverse implications do not hold, as there are clearly structures in which a symmetry sends $a$ into $b$ and yet there is no symmetry that swaps $a$ with $b$, and structures in which a symmetry swaps $a$ with $b$ but the transposition $\pi_{a b}$ is not a symmetry.

\footnotetext{
12 I don't discuss the intuitive meaning and appropriateness of any of these notions, as for the time being I am only exploring the space of logical possibilities. Distinguishing analogous concepts of symmetryindiscernibility is common in the literature (see Ladyman et al. 2012, p. 181).
} 


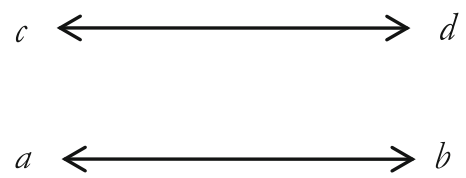

Fig. 4 A four-element class of symmetry-indiscernibility

The negations of the three types of symmetry-based indiscernibility produce three grades of discernibility in the following order of logical strength: $\neg \operatorname{Sym}_{A}(a$, $b) \Rightarrow \neg \operatorname{S}_{-} \operatorname{Sym}_{A}(a, b) \Rightarrow \neg \operatorname{SS}_{-} \operatorname{Sym}_{A}(a, b)$. We have already discussed the relations between $\neg \operatorname{Sym}_{A}(a, b)$ and absolute indiscernibility in languages $\mathcal{L}_{A}$ and $\mathcal{L}_{A}^{=}$. It remains now to place the other two symmetry-based grades of discernibility in the web of mutual logical relations with the extant grades. This can be done using the following theorems, adopted from (Ladyman et al. 2012):

Theorem 5 If $\operatorname{Rel}_{A}^{\bar{E}}(a, b)$, then $\neg \mathrm{S}_{-} \operatorname{Sym}_{A}(a, b)$.

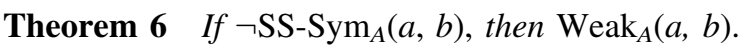

Theorem 5 follows from Ladyman, Linnebo and Pettigrew's Theorem 9.6 (2) (p. 182) by contraposition. Theorem 6 is a simple consequence of Corollary 9.4 (p. 181). Theorem 5 can be strengthened to the form of an equivalence in finite domains, but the converse of the implication in Theorem 5 has an infinite counterexample. On the other hand, a dumbbell graph is a finite counterexample to the converse of Theorem 6.

To sum up, the diagram presenting the logical relations between various possible grades of discernibility, old and new, is given below on Fig. 5 (dashed arrows indicate implications that hold in finite domains only). Now we will have to take steps to reduce its size. Slightly perversely, we will start this task by introducing yet another grade of discernibility.

\section{Witness Symmetry-Discernibility}

The notion of witness-discernibility introduced earlier is based on the assumption that a witness discerning objects $a$ and $b$ should not have a twin which would have its relations to $a$ and $b$ reversed but otherwise would be qualitatively indistinguishable from the original witness. This intuition can be spelled out in the following definition of yet another grade of discernibility, which I will call witness symmetry-discernibility $\left(\right.$ Wit-Sym $\left.{ }_{A}\right)$ :

(9) Witness symmetry-discernibility. $\operatorname{Wit}_{-\operatorname{Sym}_{A}}(a, b)$ iff there is an object $c \in$ $\operatorname{dom}(\mathcal{A})$ and a dyadic formula $\varphi$ in $\mathcal{L}_{A}$, such that.

(i) $\mathcal{A} \vDash \varphi(a, c)$ and $\mathcal{A} \not \models \varphi(b, c)$, and.

(ii) there is no object $d$ and a symmetry mapping $\pi$ such that $\pi(a)=b$, $\pi(b)=a, \pi(c)=d$, and $\pi(d)=c .^{13}$

\footnotetext{
13 It may be asked here why I didn't propose a simpler definition of symmetry-based witness discernibility in which clause (ii) would be replaced with the following condition: (iii) there is no object $d$ and symmetry $\pi$ such that $\pi(a)=b$ and $\pi(c)=d$. The answer is that, as can be quickly verified, such a notion of witness-discernibility is extensionally equivalent to $\neg \operatorname{Sym}_{\mathrm{A}}$ in finite domains.
} 


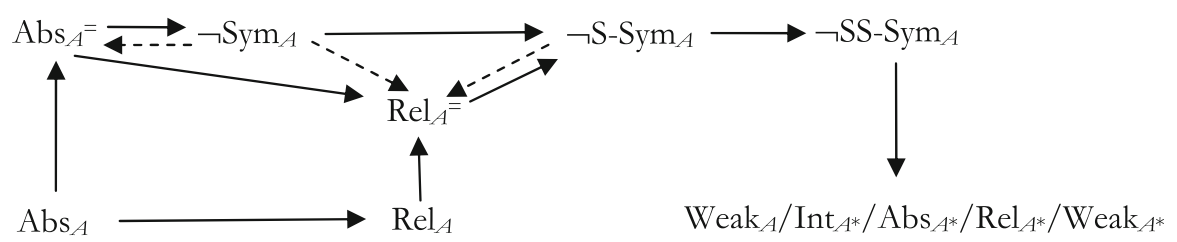

Fig. 5 Logical relations between grades of discernibility including symmetry-based ones

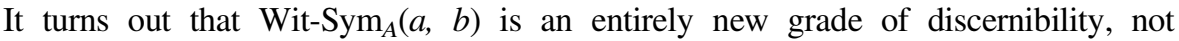
equivalent to any of the previously introduced ones, as seen in the following theorems.

Theorem 7 If $\neg \operatorname{S-Sym}(a, b)$, then $\operatorname{Wit}_{A} \operatorname{Sym}_{A}(a, b)$.

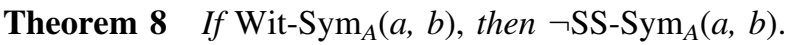

Theorem 9 The converses of Theorems 6 and 7 do not hold (not even when restricted to finite domains).

Proof of Theorem 7 We will proceed by assuming the negation of the consequent, i.e. assuming that $a$ and $b$ are not witness symmetry-discernible. Let us first consider the case in which there is no formula $\varphi$ and no element $c$ such that $\mathcal{A} \vDash \varphi(a, c)$ and $\mathcal{A} \not \models \varphi(b, c)$. This is obviously equivalent to saying that $a$ and $b$ are not even weakly discernible (they are utterly indiscernible). But utter indiscernibility implies any other grade of indiscernibility, therefore $\operatorname{S-Sym}_{A}(a, b)$ by Theorem 6 and the fact that $\mathrm{SS}_{-} \mathrm{Sym}_{A} \Rightarrow \mathrm{S}-\mathrm{Sym}_{A}$. The second case is when there is a formula $\varphi$ and an element $c$ such that $\mathcal{A} \vDash \varphi(a, c)$ and $\mathcal{A} \not \models \varphi(b, c)$. In that case there must be an element $d$ and a symmetry that swaps $c$ with $d$ and $a$ with $b$, hence $\operatorname{S}_{-} \operatorname{Sym}_{A}(a, b)$.

Proof of Theorem 8 Let us suppose that $a$ and $b$ are witness symmetry-discernible. This means that there is an object $c$ and a formula $\varphi$ such that $\mathcal{A} \vDash \varphi(a, c)$ and $\mathcal{A} \not \models \varphi(b, c)$, and such that the clause (ii) in Def. 9 holds true as well. Now we will consider two exhaustive cases: $(\alpha) a \neq c$ and $b \neq c$, ( $\beta) a=c$ or $b=c$. The assumption $(\alpha)$ of course implies that $\pi_{a b}$ is not a symmetry, given that $c$ stands in a particular relation to $a$ but not to $b$. ( $\beta$ ) Suppose that $c=a$ (the case in which $c=b$ is perfectly analogous).We have $\mathcal{A} \vDash \varphi(a, a)$ and $\mathcal{A} \not \models \varphi(b, a)$. Let us assume that the transposition $\pi_{a b}$ is a symmetry. In that case $b$ satisfies the condition expressed in (ii) and Definition 9 is violated. Thus, $\pi_{a b}$ can't be a symmetry, and

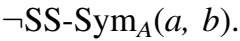

Proof of Theorem 9 A counterexample to the converse of Theorem 8 is given in Fig. 2. Objects $a$ and $b$ are not witness symmetry-discernible, as there is a symmetry which swaps $a$ with $b$ and $c$ with $d$, but $\pi_{a b}$ is not a symmetry, so $\neg \operatorname{SS}_{-} \operatorname{Sym}_{A}(a, b)$ holds. A counterexample to the converse of Theorem 7 is presented below (Fig. 6). The following permutation is clearly a symmetry: $\pi\left(c_{1}\right)=c_{2}, \pi\left(c_{2}\right)=c_{3}$, $\pi\left(c_{3}\right)=c_{4}, \pi\left(c_{4}\right)=c_{1}, \pi(a)=b, \pi(b)=a$. Thus, S-Sym ${ }_{A}(a, b)$. But $a$ and $b$ are witness symmetry-discernible. For instance $c_{1}$ is a witness to $a$ and $b$, and because there is no symmetry which would swap $c_{1}$ with any other vertex while simultaneously swapping $a$ and $b$, clause (ii) is satisfied. 


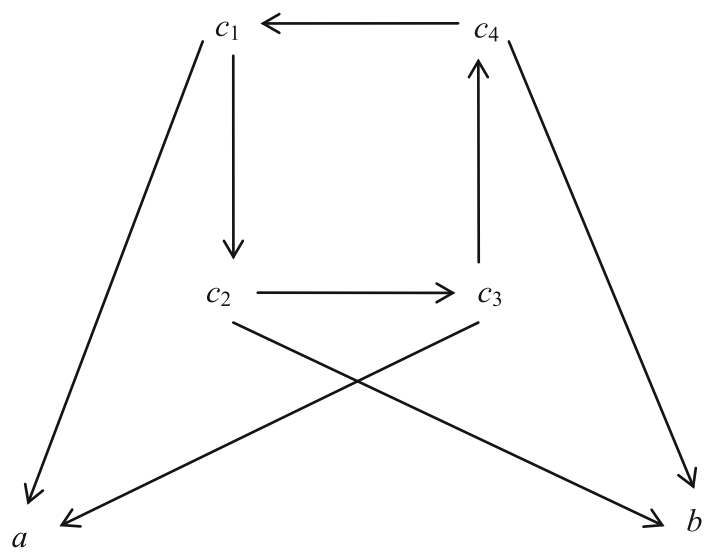

Fig. 6 A counterexample to the converse of Theorem 7

It seems evident that the relation Wit-Sym $\mathrm{S}_{A}$ represents a bona fide concept of witness-based discernibility, and thus discerning objects with the help of this relation should enable us to tell these objects apart (equivalently, make reference to one and not the other) by means of selecting a witness. On the other hand, the grade of discernibility directly following Wit-Sym ${ }_{A}$ in the hierarchy of concepts of decreasing logical strength, i.e. $\neg$ SS-Sym ${ }_{A}$, clearly does not ensure the possibility of separating discerned objects with the help of a witness. This may be confirmed by taking a quick glance at Fig. 2. Objects $a$ and $b$ are discerned by the relation $\neg$ SS-Sym , $_{A}$ as the transposition $\pi_{a b}$ is not a symmetry, and yet there is no witness in the proper sense which could differentiate $a$ from $b$ ( $c$ and $d$ are excluded, since they themselves are not discerned from each other in the proper sense). Hence, it may be hypothesized that $\mathrm{Wit}_{-\mathrm{Sym}_{A}}$ is the weakest possible notion of discernibility satisfying the requirement of ensuring the possibility of a separation of objects. Those who find this requirement compelling may now insist that no grade of discernibility weaker than Wit-Sym $A$ should be admitted as a legitimate way of discerning two objects.

But we are not done yet with the task of pruning the excess of available grades of discernibility. In the next section I will identify and critically examine a hidden premise of all discussions on discernibility which has managed to escape attention of the authors writing on this topic. Formulating this premise explicitly as a separate assumption will lead to a further reduction of the multiplicity of the remaining grades of discernibility.

\section{A Prerequisite for Discerning Two Objects}

Let us focus our attention on the well-known case of a three-element circular graph (Fig. 7). The objects in the graph are pairwise relatively discernible, as they are connected by directional arrows. Thus, they must also be categorized as witness symmetry-discernible, since this grade of discernibility is weaker than relative discernibility. But one can have legitimate doubts regarding the intuitive correctness of this assessment. To an unbiased eye the case looks like a perfect example of 


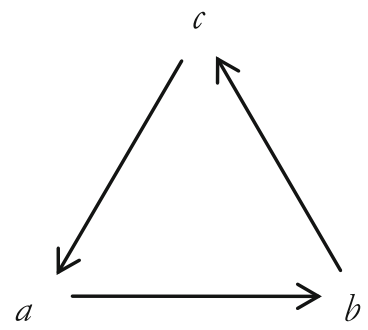

Fig. 7 A three-element circular graph

indiscernibility, if there is any. Each vertex in the triangle appears to be a perfect copy of any other vertex with respect to the place it occupies in the structure. How could there be a procedure which would single out one vertex from the entire structure?

Remaining for a while on the level of informal intuitions, we can anticipate the following reply to this challenge. It may be argued that indeed such a procedure does exist, thanks to the notion of a witness. If we consider any two out of three vertices on the diagram, it can be easily observed that one vertex from the selected pair can be discerned from the other one by the fact that there is a witness (the third vertex) which stands in different relations to both. I do not contest this intuitive assessment, but I would like to point out that the successful execution of this procedure is contingent upon an earlier separation of the entire structure into the pair of objects to be further discerned and the remaining witness. And here lies the rub: we have no reason whatsoever to believe that such a separation could be achieved on the basis of the available qualitative description of the entire relational structure. Indeed, such a separation seems to be outright impossible, since it would already achieve the goal we are pursuing, namely an effective identification of one object (i.e. the witness) against the background of the remaining elements.

We have thus managed to uncover the following implicit presupposition of all the logical reconstructions of the concept of qualitative discernibility. As the relation of discernibility is defined as a two-place relation between objects in a given structure, the assumption is that we already know which two objects we are talking about when we want to discern between them. Sometimes this assumption is indeed satisfied, but sometimes it is not, as the three-vertex case clearly shows. Thus, I propose to restrict the applicability of the notion of witness symmetry-discernibility (and any other grade of discernibility for that matter) to cases where the two objects in question are already singled out in an appropriate sense from the rest of the universe. Of course, in order to avoid the charge of question-begging, we can't use for that purpose the very notion of discernibility that we are trying to apply to discern the objects themselves. One possible solution is to resort to the more universal concept of classes of symmetryindiscernibility. The suggestion is that in order to test any relation of discernibility on a pair of objects (which, potentially, can turn out to be one and the same object), we should make sure that this pair does not belong to a symmetry-indiscernibility class containing other objects. Thus, on this approach discernment can be informally seen as a two-step procedure. In the first step we ensure that the pair of objects we are interested in can be singled out from the rest of the domain thanks to the non-existence 
of symmetries connecting these objects with some of the remaining elements of the domain. In the second step we can apply whatever discernibility relation we want to the pair selected in such a way. This informal procedure can be spelled out as follows using Wit-Sym as an example (analogous definitions can be formulated for any other grade of discernibility):

(10) If there is no class of symmetry-indiscernibility properly including $\{a, b\}$, then $\operatorname{Wit}^{-\operatorname{Sym}_{A}}(a, b)$ iff there is an object $c \in \operatorname{dom}(\mathcal{A})$ and a dyadic formula $\varphi$ in $\mathcal{L}_{A}$, such that.

(i) $\mathcal{A} \vDash \varphi(a, c)$ and $\mathcal{A} \not \models \varphi(b, c)$, and

(ii) there is no object $d$ and symmetry $\pi$ such that $\pi(a)=b, \pi(b)=a$, $\pi(c)=d$, and $\pi(d)=c$.

Let us abbreviate the condition expressed in the antecedent of (10) as $\Phi_{A}(a, b)$. I interpret the fact that $\Phi_{A}(a, b)$ is satisfied as ensuring that the pair $(a, b)$ has been effectively singled out from the background structure. In the case when $\neg \Phi_{A}(a, b)$, we can either stipulate that $a$ and $b$ are indiscernible, or assume that the two-place relation of witnessdiscernibility simply does not apply to them (it does not make sense to ask whether $a$ is discernible from $b$, since we can't even identify which $a$ and $b$ we have in mind).

Restricting the applicability of the relations of discernibility to pairs of objects satisfying condition $\Phi_{A}$ has the surprising effect of erasing the distinction between some of these relations, and therefore reducing the number of extensionally non-equivalent grades of discernibility. The following, easily provable theorems illustrate this fact:

Theorem 10 If $\Phi_{A}(a, b)$, then in finite domains $\operatorname{Rel}_{A}^{\overline{\bar{A}}}(a, b)$ iff $\operatorname{Abs}_{\bar{A}}^{\overline{\bar{A}}}(a, b)$.

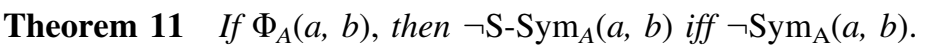

Theorem 10 states that under the assumption that $\Phi_{A}(a, b)$ relative discernibility in $\mathcal{L}_{A}^{=}$is equivalent to absolute discernibility in $\mathcal{L}_{A}^{=}$in finite domains. The right-to-left implication has already been established, so we have to prove the converse only. Let us then suppose that $\operatorname{Rel}_{A}^{\bar{E}}(a, b)$. The condition $\Phi_{A}(a, b)$ can be satisfied in two ways: either there is no symmetry connecting $a$ and $b$, or there is a symmetry which swaps $a$ and $b$. The second case is impossible, since by assumption there is a formula $\varphi$ such that $\mathcal{A} \vDash \varphi(a, b)$ and $\mathcal{A} \not \models \varphi(b, a)$, and hence no transformation that swaps $a$ with $b$ can be a symmetry. But the first case just means that $\neg \operatorname{Sym}_{A}(a, b)$, and by Theorem 2(a) $\operatorname{Abs}_{A}^{=}(a, b)$ follows in finite domains. Theorem 11 is even easier to prove. If there is a symmetry mapping $a$ into $b$ but the class of symmetry-indiscernibility containing $a$ and $b$ does not include any other element, then obviously this symmetry transposes $a$ and $b$. Hence $\operatorname{Sym}_{A}(a, b)$ implies $\operatorname{S-Sym}_{A}(a, b)$.

It may be noted that there are infinite counterexamples to the left-to-right implication of Theorem 10. One such counterexample is constructed as follows: let's take two copies of the structure consisting of integers ordered by the less-than relation- $\left\langle\mathbb{Z}_{1},<_{1}\right\rangle$ and $\left\langle\mathbb{Z}_{2},<_{2}\right\rangle$-and let's consider the structure $A=\left\langle\mathbb{Z}_{1} \cup \mathbb{Z}_{2},<\right\rangle$ such that for all $x \in \mathbb{Z}_{1}, y \in \mathbb{Z}_{2}, x<y$, while $<$ restricted to $\mathbb{Z}_{1}$ and $\mathbb{Z}_{2}$ gives $<_{1}$ and $<_{2}$, respectively. It can be easily established that $0_{1}$ and $0_{2}$ are relatively discernible by the relation $<$, and that the condition $\Phi_{A}\left(0_{1}, 0_{2}\right)$ is satisfied, since there is no 


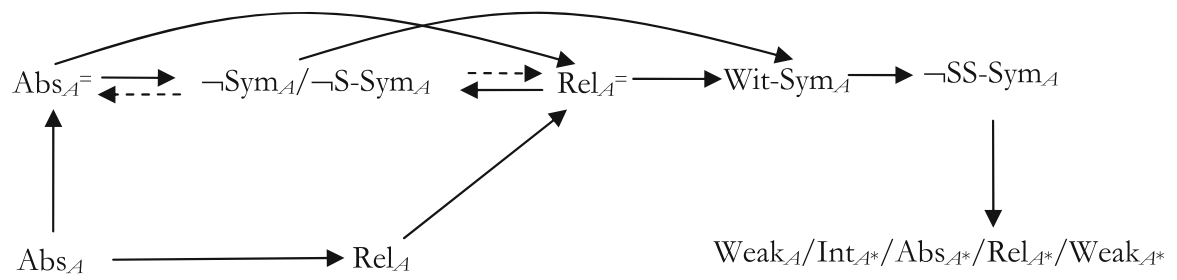

Fig. 8 Logical relations between grades of discernibility with the condition $\Phi_{A}$ in place

symmetry mapping $0_{1}$ into $0_{2}$. And yet no formula in $\mathcal{L}_{A}^{=}$absolutely discerns $0_{1}$ from $0_{2}$.

On the other hand, as all the counterexamples that we have used to falsify the implications $\operatorname{Weak}_{A}(a, b) \Rightarrow \neg \operatorname{SS}_{-} \operatorname{Sym}_{A}(a, b) \Rightarrow \operatorname{Wit}_{-} \operatorname{Sym}_{A}(a, b) \Rightarrow \neg \operatorname{S}_{-} \operatorname{Sym}_{A}(a, b)$

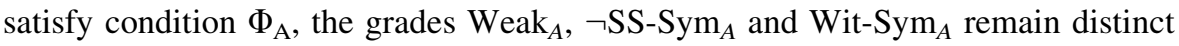
from each other (Fig. 8 depicts all the logical relations between grades of discernibility under the assumption that the condition $\Phi_{\mathrm{A}}$ is satisfied-as before, dashed arrows represent implications that hold in finite domains only). But if we agree that witness symmetry-discernibility constitutes the "upper bound" of the set of intuitively acceptable grades of discernibility, then our choice is effectively reduced to absolute discernibility in $\mathcal{L}_{A}$, relative discernibility in $\mathcal{L}_{A},{ }^{14}$ absolute discernibility in $\mathcal{L}_{A}^{=}$, and witness symmetry-discernibility. I do not wish to commit myself unconditionally to any of these four interpretations. However, at the end of this survey I would like to address one conceptual challenge that affects the weakest of the selected gradeswitness symmetry-discernibility.

\section{The Transitivity of Indiscernibility}

Ladyman, Linnebo and Pettigrew were the first to observe that relative discernibility suffers from one conceptual difficulty which does not affect the remaining two basic grades of discernibility: absolute and weak. The troublesome feature of relative discernibility is that its complement, i.e. the relation of relative indiscernibility, is not transitive, and hence cannot be an equivalence relation. This is surprising, as indiscernibility seems to be a type of identity, and therefore should possess the formal properties of an equivalence relation: reflexivity, symmetricity and transitivity. Ladyman, Linnebo and Pettigrew consider the fact that relative indiscernibility is not transitive to be a strong argument against the viability of this grade of discernibility. It is open to debate whether the non-transitivity of indiscernibility could be somehow explained away as being a result of its inherent vagueness or contextuality. I will not attempt to explore this avenue, but I would

\footnotetext{
${ }^{14}$ It can be quickly verified that $\operatorname{Rel}_{A}$ does not imply $\mathrm{Abs}_{A}$ even when the condition $\Phi_{A}$ is satisfied and the domain is finite. A simple counterexample illustrating this fact is a two-element graph in which objects $a$ and $b$ are connected by an arrow, and in addition to that an arrow links each object with itself. In this model $a$ and $b$ are relatively but not absolutely discernible in $\mathcal{L}_{A}$, and yet the condition $\Phi_{A}$ is satisfied, because there is no symmetry connecting $a$ and $b$.
} 


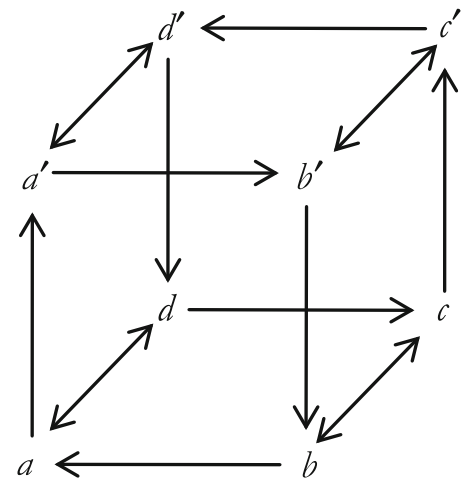

Fig. 9 A counterexample to the transitivity of indiscernibility

like to point out that two more grades of discernibility encounter the same difficulty,

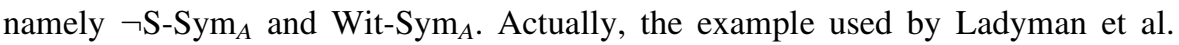
(2012, p. 183) can serve as an illustration of the violation of transitivity for both $\neg$ S-

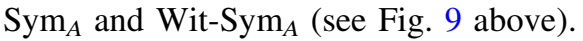

As can be quickly verified, the following permutations are symmetries of the structure depicted in the diagram: $(a c)(b d)\left(a^{\prime} c^{\prime}\right)\left(b^{\prime} d^{\prime}\right)$ and $(b c)\left(a^{\prime} d^{\prime}\right)\left(b^{\prime} d\right)\left(a c^{\prime}\right)$. This

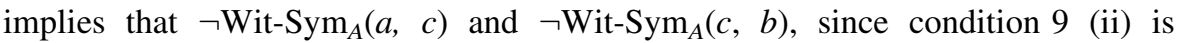
violated for all objects in the domain. But $a$ and $b$ are relatively discernible, and therefore must be witness symmetry-discernible. Hence witness symmetryindiscernibility turns out to be non-transitive.

An interesting fact, which I would like to report now, is that the restriction of the applicability of various grades of discernibility to the pairs of objects satisfying condition $\Phi_{A}$ has the effect of securing the validity of transitivity, albeit in a semivacuous form. That is, transitivity of the complement of any relation of discernibility can never be violated, because we can't find three distinct objects $a, b, c$ such that $\Phi_{A}(a, b), \Phi_{A}(b, c)$, and $a$ is indiscernible from $b$ while $b$ is indiscernible from $c$ (in any sense of indiscernibility stronger than $\left.\operatorname{Sym}_{A}\right)$. From the assumption that pairs $(a, b)$ and $(b, c)$ are indiscernible it follows that $\operatorname{Sym}_{A}(a, b)$ and $\operatorname{Sym}_{A}(b, c)$. In that case the truth of $\Phi_{A}(a, b)$ implies that $\{a, b\}$ is a separate class of symmetry-indiscernibility, and therefore there can't be a distinct object $c$ such that $\Phi_{A}(b, c)$. On the other hand, it is impossible to find a counterexample to transitivity using only two objects $a$ and $b$, since all relations of indiscernibility are reflexive. Thus, as long as we limit ourselves to pairs satisfying $\Phi_{A}$, no problem of transitivity arises.

This result can be easily repeated in case we interpret a given relation of indiscernibility as including all pairs $(a, b)$ for which $\neg \Phi_{A}(a, b)$. That is, it can be quickly verified that the relation $\neg \Phi_{A}(a, b) \vee \neg D_{A}(a, b)$, where $D_{A}$ is any grade of discernibility weaker than $\neg \operatorname{Sym}_{A}$, is transitive. To see that, let us notice first that the relation $\neg \Phi_{A}(a, b)$ is itself transitive (this follows directly from the definition). Thus, we have to consider two cases only: (i) $\neg \Phi_{A}(a, b)$ and $\neg D_{A}(b, c)$, (ii) $\neg D_{A}(a, b)$ and $\neg D_{A}(b, c)$. Thanks to the assumption that $\neg D_{A}(a, b) \rightarrow \operatorname{Sym}_{A}(a, b)$, both in case (i) and in case (ii) it can be inferred that $a, b, c$ belong to the same class of indiscernibility. This can already secure the conclusion that $\neg \Phi_{A}(a, c)$ if only $a, b$, 
$c$ are three distinct objects. But if some of these objects are in fact identical, the condition of transitivity is satisfied trivially, as we have already noted. Hence, the stated fact is proven.

\section{Conclusion}

I have used two main tools to trim the excess of available grades of discernibility. One of them was the intuition that the primary purpose of discerning objects is to be able to tell them apart. This intuition finds its formal reconstruction in the notion of witness symmetry-discernibility as the weakest acceptable grade of discernibility. The other tool was the assumption that before we can even start discerning any two objects we have to find a way to single them out from the rest of the domain. Combining these two tools we have achieved a dramatic reduction of the number of acceptable grades of discernibility. If we limit ourselves to finite domains and admit identity in the vocabulary of our language, then the choice is between absolute discernibility and witness symmetrydiscernibility only. Admitting infinite models gives us two additional grades of discernibility: symmetry-discernibility and relative discernibility. Those who find the use of the identity symbol objectionable may wish to use the concepts of absolute and relative discernibility in the language $\mathcal{L}_{A}$ rather than $\mathcal{L}_{A}^{=}$. But they have to accept the fact that these two concepts do not coincide even in models with finite domains.

How relevant to the current discussions on the status of objects in quantum mechanics and relativity is the logical analysis carried out in this paper? An exhaustive answer to this question would probably require an altogether new paper. However, some immediate metaphysical and methodological consequences are relatively easy to draw. As I mentioned on several occasions in this paper, the main focus of the metaphysical debates regarding the status of elementary particles and spatiotemporal points has been on the concept of weak discernibility. Given the well-known fact that the entities postulated in our most fundamental physical theories are weakly discernible by appropriate physically meaningful relations, it is often argued that this fact is sufficient to grant the fundamental physical entities the status of objects possessing some form of quality-based individuality (with no need of resorting to haecceities or other metaphysically suspicious notions). One of the main lessons of this paper is that this argument is too hasty. Individuality presupposes the possibility of making reference to separate objects, and-as I tried to argue above-not all grades of discernibility guarantee that. Arguably, the weakest grade of discernibility that can help us tell two objects apart in the way which makes it possible to refer to any one of them separately is witness symmetrydiscernibility. But it is straightforward to observe that the permutation invariance of systems of particles of the same type in quantum mechanics, and the existence of non-trivial symmetries of spacetime in GR, prevent the fundamental entities of these theories from being discernible by the relation Wit-Sym $\mathrm{W}_{A}$. The relation of weak discernibility holding between two particles of the same type, or between two spatiotemporal points, can ensure that the number of objects is indeed two, but falls short of separating them in the way individuals are supposed to be separable. 
The additional restriction of discernibility to pairs of objects which don't belong to three-or-more-element classes of symmetry-indiscernibility (as suggested in Sect. 6) further compounds the situation of the defenders of the qualitative individuality of fundamental entities in physics. For presently no relation of discernibility (not even weak discernibility) seems to be applicable to these objects, since they certainly belong to enormous classes of symmetry-indiscernibility (think about all the electrons in the universe forming one incredibly large class of indiscernibility). Thus, it looks like those who insist that our best physical theories postulate entities whose metaphysical status is that of non-individuals receive an additional argument in favor of their claim. One possible reaction to this development is to accept the above verdict and try to develop the best logical theory of such non-individuals (for instance in the form of either quasi-set or quaset theory; for a survey of these options see e.g. Dalla Chiara et al. 1998). But an alternative option-which I urge but have no space to adequately argue for-is to take a step back and reconsider the origin of the whole quandary. My subsequent remarks about this option may be seen as rather nebulous, but I hope that the readers will understand that I could not possibly explain this complicated matter to their full satisfaction in the remainder of this paper.

At the bottom of the metaphysical problems with fundamental physical entities lies the Absolute Indiscernibility Thesis. Restricting ourselves to the case of quantum mechanics, we can spell it out in the form of the claim that, due to the Symmetrization Postulate, no two particles of the same type can ever be absolutely discerned by their properties (whether monadic or relational). The arguments in favor of this claim given in the literature (see footnote 2 for references) rely on the labeled tensor product Hilbert space formalism, to use Teller's famous expression (Teller 1995, p. 20). Some commentators, including Teller himself, argue that this formalism is not metaphysically neutral, as it seems to presuppose the existence of primitive identities expressed in the labels. Thus the question arises whether it is possible to formulate the problem of absolute discernibility in a more neutral framework. One option is to use the Fock space formalism, but another, perhaps less radical, is to retain the tensor product formalism but limit the acceptable physical quantities to the symmetric ones (in which case the labels can assume the role of a harmless formal tool with no deeper meaning). I have no space to go into any details here, but it may be argued that in such a case particles of the same type can, in certain experimental situations, be claimed to be absolutely discernible by their adequately formalized properties. Interested readers can find a more detailed though still preliminary discussion of this controversial claim in my (Bigaj 2015). If this argument is correct (and this is a big "if"), then in a surprising twist quantum particles may be "rehabilitated" as objects capable of being picked out and referred to individually, and not only collectively in the form of aggregates.

Acknowledgments I would like to thank Ewa Bigaj for her help at various stages of writing this article. I am particularly grateful to two anonymous referees for their detailed comments which helped me express my thoughts more clearly. The work on this article was supported by the Marie Curie IOF 2012 Grant No. 328285. 
Open Access This article is distributed under the terms of the Creative Commons Attribution License which permits any use, distribution, and reproduction in any medium, provided the original author(s) and the source are credited.

\section{References}

Bigaj, T. (2015). Exchanging quantum particles. In P.E. Bour, G. Heinzmann, W. Hodges, \& P. Schroeder-Heister (eds.), 14th CLMPS 2011 Proceedings, Philosophia Scientiae (Vol. 19, issue 1) (forthcoming).

Butterfield, J. (1993). Interpretation and identity in quantum theory. Studies in History and Philosophy of Science, 24, 443-476.

Caulton, A., \& Butterfield, J. (2012). On kinds of indiscernibility in logic and metaphysics. British Journal for the Philosophy of Science, 63, 27-84.

Dalla Chiara, M. L., Giuntini, R., \& Krause, D. (1998). Quasiset theories for microobjects: A comparison. In E. Castellani (Ed.), Interpreting bodies: Classical and quantum objects in modern physics (pp. 142-152). Princeton: Princeton University Press.

Dieks, D., \& Versteegh, M. (2008). Identical quantum particles and weak discernibility. Foundations of Physics, 38, 923-934.

Dorato, M., \& Morganti, M. (2013). Grades of individuality. A pluralistic view of identity in quantum mechanics and in the sciences. Philosophical Studies, 163, 591-610.

French, S., \& Krause, D. (2006). Identity in physics. Oxford: Oxford University Press.

French, S., \& Redhead, M. (1988). Quantum physics and the identity of indiscernibles. British Journal for the Philosophy of Science, 39, 233-246.

Hawley, K. (2006). Weak discernibility. Analysis, 66, 300-303.

Hawley, K. (2009). Identity and indiscernibility. Mind, 118, 101-119.

Huggett, N., \& Norton, J. (2013). Weak discernibility for quanta, the right way. British Journal for the Philosophy of Science. doi:10.1093/bjps/axs038.

Ketland, J. (2011). Identity and indiscernibility. The Review of Symbolic Logic, 4(2), 171-185.

Ladyman, J., \& Bigaj, T. (2010). The principle of the identity of indiscernibles and quantum mechanics. Philosophy of Science, 77, 117-136.

Ladyman, J., Linnebo, O., \& Pettigrew, R. (2012). Identity and discernibility in philosophy and logic. The Review of Symbolic Logic, 5, 162-186.

Linnebo, O., \& Muller, F. A. (2013). On witness-discernibility of elementary particles. Erkenntnis, 78, 1133-1142.

Muller, F. A., \& Saunders, S. (2008). Discerning fermions. British Journal for the Philosophy of Science, 59, 499-548.

Muller, F. A., \& Seevinck, M. P. (2009). Discerning elementary particles. Philosophy of Science, 76, 179-200.

Quine, W. V. O. (1976). Grades of discriminability. Journal of Philosophy, 73, 113-116.

Saunders, S. (2003). Physics and Leibniz's principles. In K. Brading \& E. Castellani (Eds.), Symmetries in physics: Philosophical reflections (pp. 289-307). Cambridge: Cambridge University Press.

Saunders, S. (2006). Are quantum particles objects? Analysis, 66, 52-63.

Teller, P. (1995). An interpretive introduction to quantum field theory. Princeton: Princeton University Press.

van Fraassen, B., \& Peschard, I. (2008). Identity over time: Objectively, subjectively. Philosophical Quarterly, 58, 15-35.

Wüthrich, C. (2009). Challenging the spacetime structuralist. Philosophy of Science, 76, 1039-1051. 